\title{
A Virtual Sensor for Needle Deflection Estimation during Soft-Tissue Needle Insertion
}

\author{
Thomas Lehmann ${ }^{1}$, Carlos Rossa $^{1}$, Nawaid Usmani ${ }^{2}$, Ronald Sloboda ${ }^{2}$ and Mahdi Tavakoli ${ }^{1}$
}

\begin{abstract}
A tissue-independent model to estimate needle deflection during insertion in soft tissue is presented in this paper. A force/torque sensor is connected to the needle base in order to measure forces and moments during insertion due to needle deflection. A static mechanical model, which is based on the Euler-Bernoulli beam equation and the balance of forces applied by the tissue onto the needle takes these force and moment measurements as input. The needle tip deflection can then be calculated based on the beam model undergoing these forces. Three different needle-tissue interaction models are presented. Their estimation performance is evaluated and experimentally compared by carrying out insertion experiments into phantom tissue. The experimental results show a precise estimate of needle tip deflection for a novel virtual sensor introduced in this work. The main advantage of this virtual sensor approach is that measurements obtained from the force/torque sensor are the only necessary model inputs. Furthermore, the approach does not rely on ultrasound or other image-based needle observation techniques. This makes the virtual sensor suitable for real-time feedback of needle tip deflection.
\end{abstract}

\section{INTRODUCTION}

Needle insertion into soft tissue is performed in multiple medical applications such as biopsy and cancer treatment. Needles are used to plant radioactive seeds in soft tissue as done in interstitial prostate brachytherapy. In prostate brachytherapy, the implanted radioactive seeds eliminate cancer cells from close proximity. Depending on the procedure, the needles either need to be steered to their target locations or kept on a straight path in order to maximize targeting accuracy and thus treatment efficiency.

The tip of a standard brachytherapy needle is beveled, which causes asymmetric forces enacted by the tissue onto the needle tip. Due to this imbalance, the needle deflects from its intended straight path during insertion. The deflection causes displacement of the seeds from their desired locations, which leads to inefficient and misplaced radiation dosages. The goal of robot-assisted needle insertion is therefore to minimize the needle deflection by using the aforementioned force asymmetry to steer the needle back to its straight path

This work was supported by the Natural Sciences and Engineering Research Council (NSERC) of Canada under grant CHRP 446520, the Canadian Institutes of Health Research (CIHR) under grant CPG 127768, and by the Alberta Innovates - Health Solutions (AIHS) under grant CRIO 201201232.

${ }^{1}$ Thomas Lehmann, Carlos Rossa and Mahdi Tavakoli (Corresponding Author) are with the Department of Electrical and Computer Engineering, University of Alberta, AB, Canada T6G 2V4. E-mail: \{lehmann, rossa, mahdi.tavakoli\}@ualberta.ca

${ }^{2}$ Ronald Sloboda and Nawaid Usmani are with the Cross Cancer Institute and the Department of Oncology, University of Alberta, Edmonton, AB, Canada T6G 1Z2. E-mail: \{ron.sloboda, nawaid.usmani\}@albertahealthservices.ca. e.g. by rotating the needle by $180^{\circ}$ about its longitudinal axis More generally, for any on-line needle steering or control algorithm, needle deflection feedback is necessary.

Needle deflection feedback is often obtained by directly observing the needle position during insertion through imaging modalities such as ultrasound (US) or X-Ray [1], [2], [3]. However, these modalities are limited in resolution and require real-time processing to automatically track the needle. The very acquisition of US or X-Ray images in a real-time fashion may also not be straightforward in current operating rooms. Other solutions to estimate needle deflection are often sought in physically modelling the needle-tissue interaction in order to predict the needle deflection. To this end, several needle-tissue physical models have been proposed [4], [5], [6], [7]. Although such models have been demonstrated to be able to predict needle deflection, they require as input the hard-to-characterize needle-tissue interaction parameters such as interaction force profiles and/or tissue stiffness.

We propose in this paper an improved method for the estimation of needle tip deflection in real-time based only on forces and moments measured at the needle base by a force/torque $(\mathrm{F} / \mathrm{T})$ sensor. This work is based on a concept introduced in [8], [9]. In [8], only the tip force necessary to cut the tissue is considered and other tissue reactions enacted onto the needle are assumed to be negligible. Here, we further extend these models by considering tissue reaction along the needle caused by its compression and cutting. We consider three different needle-tissue models based on different load distributions along the needle shaft. The resulting novel needle deflection model represents an improvement over previous work [8], [9] due to revised force distribution profiles.

Force and moment measured at the needle base during insertion are the only inputs to the proposed deflection model. Thus, the model does not require the explicit measurement of tissue parameters. Based on a needle-tissue interaction model, the force and moment measurements are related to the reaction forces applied by tissue along the needle. The needle, which represents a cantilever beam, is modelled by the Euler-Bernoulli beam equation, which is, as mentioned, exposed to the needle-tissue interaction loads. The model is considered in the static domain, which means that equilibrium conditions for forces and moments apply.

The layout of this paper is as follows. In Section II, we introduce three different needle-tissue interaction models. For each model, the relation between measured force/moment are related to the needle deflection. Subsequently, in section III, the experimental setup used to conduct needle 
insertion experiments in two different phantom tissues is shown. Furthermore, we provide a comparison between the measured and estimated needle deflection, in order to assess and compare the accuracy of the three models. The results show that the best model is able to estimate the needle deflection with a mean absolute error (MAE) below $1 \mathrm{~mm}$ for an insertion depth of up to $120 \mathrm{~mm}$.

\section{NeEdle Deflection Models}

This section introduces the proposed models for needletissue interaction. The underlying methodology, which relates the force/moment equilibrium in the static case to the deflection of a beam is introduced. In the proposed model, there are two steps for estimating the deflection namely 1) writing the force and moment balance equations for the modelled loads along the needle and 2) subjecting the deflection model of the cantilever beam exposed to these loads. These steps are described in the following two subsections. Needle deflection at each insertion step can then be calculated.

We consider in this paper two different distributions for the force exerted by the tissue onto the needle, namely uniform and triangular distributions, which serve as the basis for our three different deflection models. These considerations are further explained in the following subsections.

\section{A. Model 1: Two Triangularly Distributed Loads}

This model was introduced in our previous work [9]. It is based on the assumption that two triangularly distributed loads act along the portion of the needle that is in contact with tissue. The model is shown in Fig. 1(a).

A needle is inserted into tissue. Its projection along the straight needle axis $(y)$ is called $L$. Also, $l_{\text {out }}$ and $l_{\text {in }}=L-l_{\text {out }}$ are the projections needle portions that are outside and inside of tissue, respectively. We consider the difference between the physical needle length and its projection along $y$ as it bends (i.e. $L$ ) to be negligible. The resultant force and moment induced by the needle-tissue interaction forces at point $A$ are $F_{R}$ and $M_{R}$, respectively. $F_{R}$ and $M_{R}$ are measured by a force sensor. The distance between the needle base and the point $A$ is $c$. The needle tip deflection is called $\delta_{\text {tip }}$.

As the needle bends while being inserted into tissue, it compresses the tissue below the needle as shown in Fig. 1(a). The force applied by the compressed tissue is assumed to form a triangularly distributed load $q_{1, t}$. The assumption is that as the needle bends, it increasingly compresses tissue below the needle. Therefore, it is reasonable to assume that the tissue applies the highest reaction force at the needle entry point and the least reaction force at the needle tip [8]. Consequently, $q_{1, t}$ is assumed to be at its maximum at the needle entry point and at its minimum at the needle tip. According to beam theory [10], $q_{1, t}$ can be replaced by a point load $F_{1, t}$ at the centroid of $q_{1, t}$ at point B (see Fig. 1(a)). The centroid and thus $F_{1, t}$ is located at $1 / 3$ of the inserted needle length $l_{\text {in }}$. Above the needle, however, the triangular load distribution $q_{2, t}$ is assumed because the needle stores potential energy in its bent state, which results in a pressure

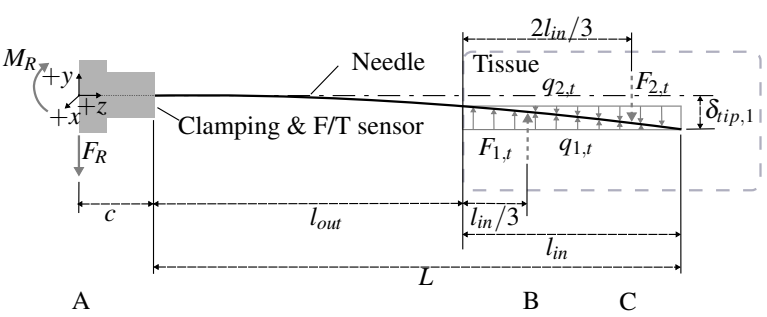

(a) Model 1: Two triangularly distributed loads.

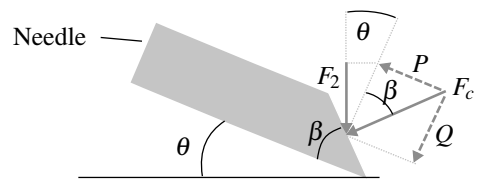

(b) A small needle portion at the tip showing

forces acting at the needle tip

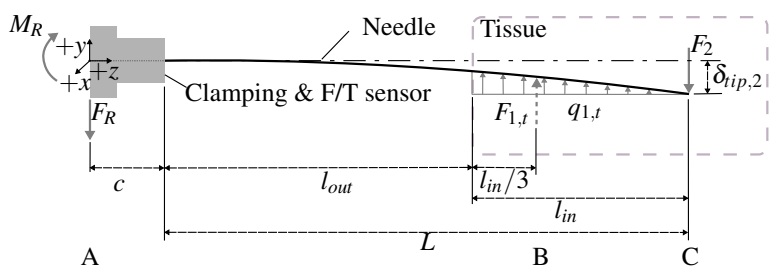

(c) Model with above force $\left(F_{2}\right)$ at the tip.

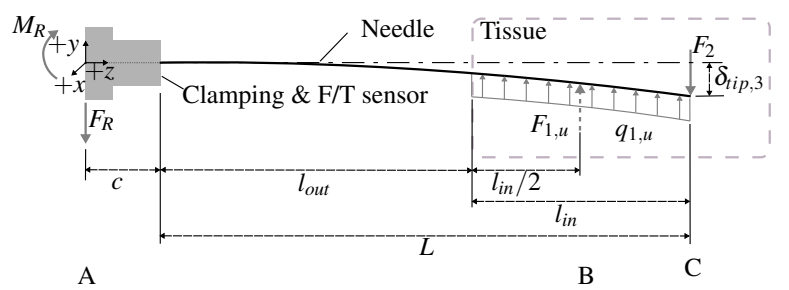

(d) Model with uniformly distributed load below.

Fig. 1. Needle-tissue interaction model.(a) Forces acting on the needle tip during insertion. $F_{c}$ is the force normal to the needle bevel, $Q$ and $P$ are the transverse and axial component of $Q$ respectively and $F_{2}$ is the projection of $Q$ along $y . \beta$ is the bevel angle and $\theta$ is the deflection slope.(b) The assumed tissue loads acting on the needle are $q_{1, t}$ and $q_{2, t} . q_{1, t}$ and $q_{2, t}$ are triangularly distributed loads that can be replaced by the point load $F_{1, t}$ and $F_{2, t}$, respectively. The measured force at the point $\mathrm{A}$ are $F_{R}$ and $M_{R}$. The distance between $\mathrm{A}$ and the needle base is $c$. The projection along $z$ of the total needle length, of portion of the needle inside and outside the tissue respectively are $L, l_{\text {in }}$ and $l_{\text {out }}$. The tip deflection is called $\delta_{\text {tip }}$. (c) A proposed model variant of (a) with $q_{2, t}$ changed to the point load $F_{2}$ acting at the needle tip. (d) The load $q_{1, t}$ in (c) was changed to the uniformly distributed load $q_{1, u}$.

applied onto the tissue in the upward direction, which causes a downwards pointing tissue reaction $\left(q_{2, t}\right)$. Here, the point load $F_{2, t}$ replacing $q_{2, t}$ is placed at $2 / 3$ of $l_{i n}$.

In order to relate the measured force and moment $F_{R}$ and $M_{R}$ to the loads $F_{1, t}$ and $F_{2, t}$ acting along the needle, the force and moment balance equations need to be established. The balance of moments acting at point $A$ gives

$$
-M_{R}+F_{1, t}\left(l_{1}+c\right)-F_{2, t}\left(l_{2}+c\right)=0
$$

Also at point $A$, the balance of forces yields

$$
-F_{R}+F_{1}-F_{2}=0
$$


in (1),

$$
\begin{aligned}
& l_{1}=l_{\text {out }}+l_{\text {in }} a \\
& l_{2}=l_{\text {out }}+l_{\text {in }} b
\end{aligned}
$$

where $a=1 / 3, b=2 / 3$ and $c$ is the distance between the needle base and the origin of the force sensor's frame as shown in Fig. 1. The unknown forces $F_{1, t}$ and $F_{2, t}$ can now be found by using (1) and (2):

$$
\begin{aligned}
& F_{1, t}=\frac{F_{R}\left(l_{2}+c\right)-M_{R}}{l_{1}-l_{2}} \\
& F_{2, t}=-F_{R}-F_{1, t}
\end{aligned}
$$

The deflection generated by $F_{1, t}$ and $F_{2, t}$ can now be calculated by modelling the needle as a cantilever beam undergoing these loads. The needle deflection is calculated in the static case in each discretized insertion step. Steps will be one after another starting from the unbent needle.

The deflection model is a static cantilever beam model based on the Euler-Bernoulli beam theory. For a beam experiencing an overall load of $q(z)$, also known as forcing term, along its longitudinal axis $z$ with respect to the needle's base, the deflection $v(z)$ in $y$ direction is governed by

$$
\frac{d^{2}}{d z^{2}}\left(E I \frac{d^{2} v(z)}{d z^{2}}\right)=q(z)
$$

where $E$ and $I$ are the Young's modulus and area moment of inertia of the needle.

As we have two loads acting on the needle (see Fig. 1), in order to obtain the overall tip deflection, the deflections caused by each force are separately calculated and superimposed.

The deflections $\left(\delta_{1, t}\right.$ and $\left.\delta_{2, t}\right)$ caused by the forces $F_{1, t}$ and $F_{2, t}$ can be computed as:

$$
\begin{aligned}
& \delta_{1, t}=\frac{\left(3 L-l_{1}\right) l_{1}^{2}}{6 E I} F_{1, t} \\
& \delta_{2, t}=\frac{\left(3 L-l_{2}\right) l_{2}^{2}}{6 E I} F_{2, t}
\end{aligned}
$$

where $l_{1}$ is defined by (3) and $l_{2}$ by (4).

Finally, the resulting deflection $\delta_{t i p, 1}$ at the needle tip is [10]:

$$
\delta_{\text {tip }, 1}=\delta_{1, t}+\delta_{2, t}
$$

\section{B. Model 2: A Triangularly Distributed Load and Cutting- Induced Point Load}

This model considers the cutting-induced point load at the needle tip $F_{2}$. The origin of $F_{2}$ is shown in Fig. 1(b), which illustrates the forces acting at the needle tip during insertion into tissue. As the needle is inserted, the force $F_{c}$ is applied by tissue at the needle tip, in a direction perpendicular to the needle bevel surface. The force $F_{c}$ can be decomposed in its transverse component $Q$ and axial component $P$ which depend on the needle bevel angle $\beta$. The transverse force component $Q$ causes the needle to bend as it is inserted into the tissue. The influence of the axial force component $P$ on the deflection is neglected as it predominantly causes very small longitudinal compression of the needle. The relation

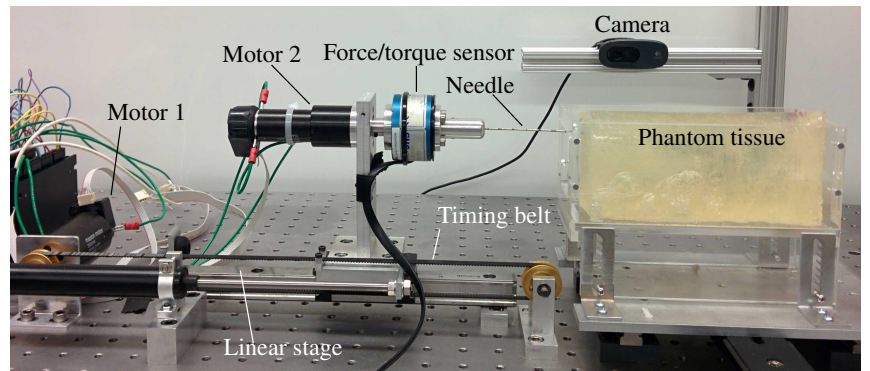

Fig. 2. The experimental setup for performing needle insertions. A DC motor (Motor 1) provides the linear motion to insert the needle into the tissue. A second motor (Motor 2) attached to the needle base is able to rotate it around its axis during insertion (not used in this paper). The forces at the needle base are measured by a force/torque sensor. Images of the needle inside tissue are recorded by a Logitech C270 camera.

between $F_{2}$ and $Q$ is given by $F_{2}=Q \cos \theta$ where $\theta$ is the needle bending angle at its tip.

As the needle penetrates into the tissue and deflects in the direction of $F_{2}$, it applies pressure onto the tissue. As in Model 1, a triangularly distributed load is considered below the needle. The force distribution model is shown in Fig. 1(c). In this model, $q_{1, t}$ is again replaced by a point load and (8) can be applied to calculate the tip deflection component $\delta_{1, t}$. The deflection $\delta_{2}$ caused by $F_{2}$ is:

$$
\delta_{2}=\frac{L^{3}}{3 E I} F_{2}
$$

The resulting deflection $\delta_{t i p, 2}$ is the combination of (8) and (11):

$$
\delta_{t i p, 2}=\delta_{1, t}+\delta_{2}
$$

\section{Model 3: A Uniformly Distributed Load and Cutting- Induced Point Load}

In this model we consider the cutting force related load for the portion of the tissue above the needle $F_{2}$ as presented in Model 2. In contrast to Model 2, in this model the reaction of the compressed tissue below the needle (see Fig. 1(d)) is assumed to have a uniform distribution along the portion of the needle that is inside the tissue [6]. This reaction is modelled as a load per length $q_{1, u}$. According to beam theory [10], the uniformly distributed load $q_{1, u}$ can again be replaced by the point load $F_{1, u}$ at the centroid of $q_{1, u}$, which is at point B. For a uniformly distributed load, the point B is placed at $1 / 2$ of $l_{\text {in }}$.

The deflection caused by $F_{1, u}$ is

$$
\delta_{1, u}=\frac{\left(3 L-l_{1}\right) l_{1}^{2}}{6 E I} F_{1, u}
$$

where $l_{1}$ is defined by (3) with $a=1 / 2$.

The total tip deflection $\delta_{t i p, 3}$ is given by (11) and (13):

$$
\delta_{t i p, 3}=\delta_{1, u}+\delta_{2}
$$




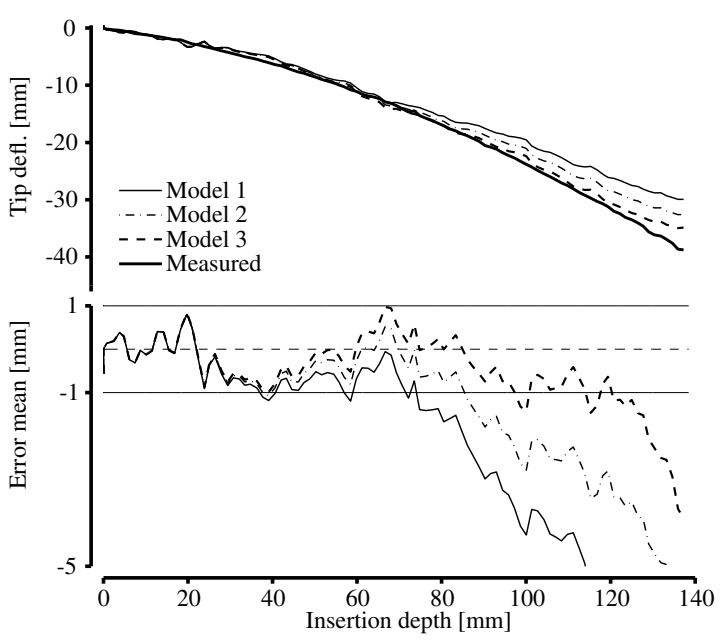

(a) Tissue 1, insertion velocity: $20 \mathrm{~mm} / \mathrm{s}$.

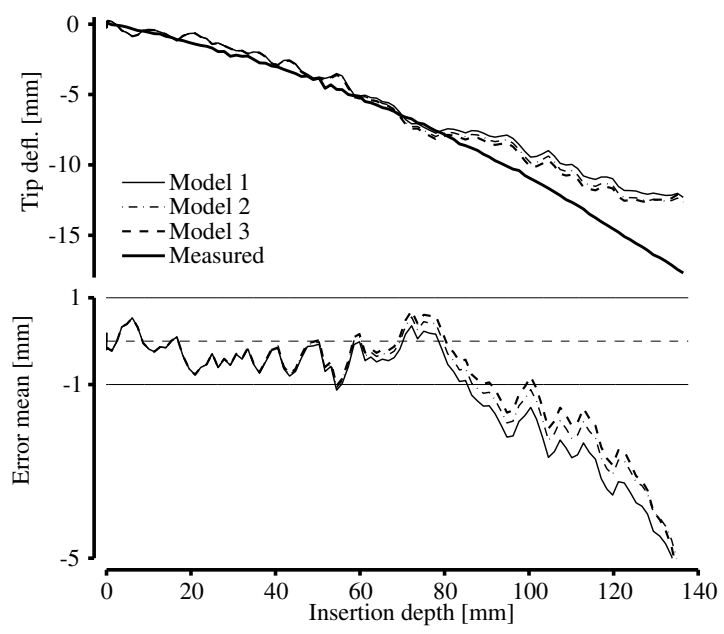

(c) Tissue 2, velocity: $20 \mathrm{~mm} / \mathrm{s}$.

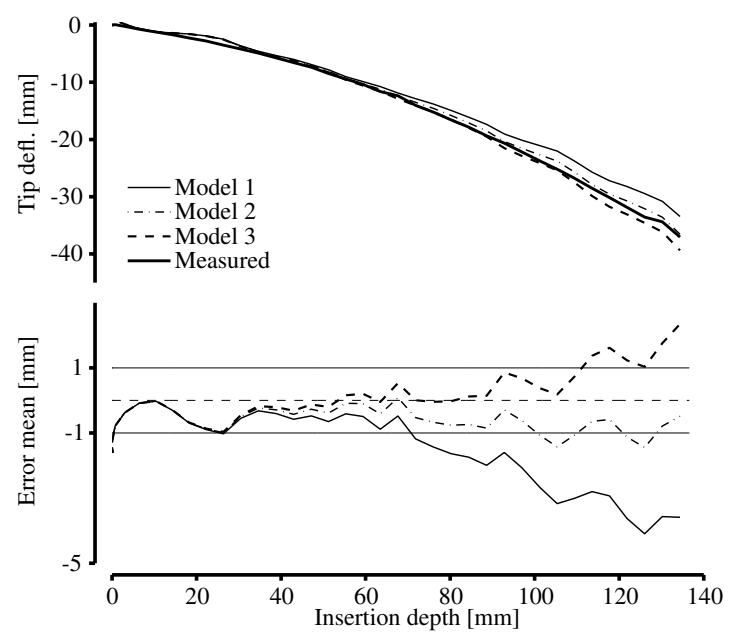

(b) Tissue 1, insertion velocity: $60 \mathrm{~mm} / \mathrm{s}$.

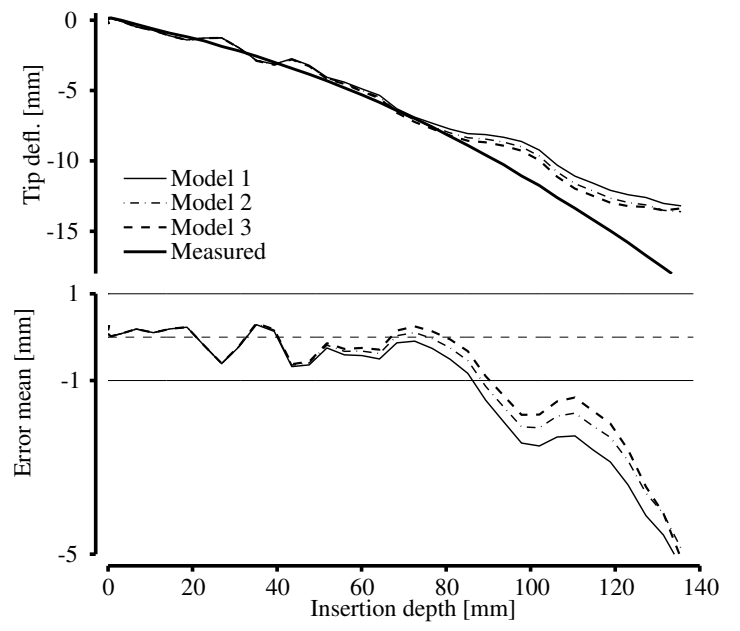

(d) Tissue 2, velocity: $60 \mathrm{~mm} / \mathrm{s}$.

Fig. 3. The mean curves of three runs with each of two tissue samples and two insertion velocities. The error plot shows the relative error between measured and estimated deflection $(\delta-\hat{\delta})$. The measured deflection $\delta$ is calculated based on camera images.

\section{EXPERIMENTAL RESULTS}

The experimental setup used to perform insertion experiments is the 2 Degree-of-Freedom (DOF) prismatic-revolute robotic system shown in Fig. 2. The needle, which represents the end-effector of the robot, can be translated along and rotated about its longitudinal axis. The translational motion is guided by a linear stage. The linear stage's carriage is coupled to a timing belt, which is driven by a DC motor (RE40, Maxon Motor AG, Sachseln, Switzerland). The rotational motion is not utilized during the experiments presented in this work. The rotational motor's shaft carries a 6 DOF force/torque transducer (50M31A3-I25, JR3 Inc., Woodland, CA, USA) to record the 2 DOF of forces and torques, which are the model inputs. The sensor's remaining 4 DOF are not used. Constant velocity insertions are facilitated using a PI controller.

Needle tip deflection measurements for model validation are carried out by analyzing images of the needle inserted inside phantom tissue. Images are continuously taken throughout insertion with a framerate of $30 \mathrm{~Hz}$. A Logitech
C270 camera is used for image acquisition. As only one camera is used to record the needle inside semi-transparent phantom tissue, the needle is steered, such that the plane of deflection is parallel to the image plane. In order to detect the needle tip in each image, first the images are pre-conditioned by contrast enhancement and noise filtering via a Wiener low-pass filter [11]. Then, a Sobel operator [11] is applied to detect the needle edges. The detected needle contour is then filled and analyzed by the RANSAC algorithm [12], which fits a $2^{\text {nd }}$ order polynomial onto the points representing the needle. In order to find the needle tip, the arc length of the identified $2^{\text {nd }}$ order polynomial $p(x)$ is calculated iteratively while increasing $x$, the horizontal pixel coordinate. The current insertion depth $l_{i n}$, which is known independently, is compared to the arclength of $p(x)$. If the arclength of $p(x)>=l_{\text {in }}$, then $[x, p(x)]$ is the needle tip. Note that $l_{\text {in }}$ can be obtained from the insertion robot. Fig. 5 shows the polynomial fit and the found tip as a cross for a sample insertion. A flowchart showing the detection algorithm can be found in Fig. 4. 
TABLE I

MeAn Absolute ERror (MAE), AbSOlute mean $(|\overline{\boldsymbol{\delta}}|)$ AND STANDARd ERror $(\sigma / \sqrt{n})$ FOR ALL MOdels AT TWO INSERTION DEPTHS.

\begin{tabular}{|c|c|c|c|c|c|c|c|c|c|c|c|c|c|}
\hline \multirow{3}{*}{ Model \# } & \multirow{3}{*}{ Insertion depth } & \multicolumn{6}{|c|}{ Tissue 1} & \multicolumn{6}{|c|}{ Tissue 2} \\
\hline & & \multicolumn{3}{|c|}{$20 \mathrm{mms}^{-1}$} & \multicolumn{3}{|c|}{$60 \mathrm{mms}^{-1}$} & \multicolumn{3}{|c|}{$20 \mathrm{mms}^{-1}$} & \multicolumn{3}{|c|}{$60 \mathrm{mms}^{-1}$} \\
\hline & & MAE & $|\bar{\delta}|$ & $\sigma / \sqrt{n}$ & MAE & $|\bar{\delta}|$ & $\sigma / \sqrt{n}$ & MAE & $|\bar{\delta}|$ & $\sigma / \sqrt{n}$ & MAE & $|\bar{\delta}|$ & $\sigma / \sqrt{n}$ \\
\hline 1 & $60 \mathrm{~mm}$ & 0.56 & 10.49 & 0.34 & 0.38 & 10.81 & 0.27 & 0.36 & 5.17 & 0.84 & 0.28 & 4.88 & 0.12 \\
\hline 2 & $60 \mathrm{~mm}$ & 0.46 & 10.91 & 0.36 & 0.47 & 11.25 & 0.28 & 0.34 & 5.27 & 0.85 & 0.26 & 4.98 & 0.13 \\
\hline 3 & $60 \mathrm{~mm}$ & 0.39 & 11.23 & 0.37 & 0.54 & 11.59 & 0.29 & 0.34 & 5.35 & 0.86 & 0.25 & 5.05 & 0.14 \\
\hline 1 & $120 \mathrm{~mm}$ & 1.58 & 26.14 & 0.56 & 0.79 & 28.34 & 0.59 & 0.87 & 11.25 & 0.38 & 0.79 & 12.17 & 0.74 \\
\hline 2 & $120 \mathrm{~mm}$ & 0.96 & 28.46 & 0.60 & 0.39 & 30.86 & 0.67 & 0.75 & 11.73 & 0.40 & 0.64 & 12.73 & 0.83 \\
\hline 3 & $120 \mathrm{~mm}$ & 0.49 & 30.65 & 0.65 & 0.89 & 33.25 & 0.76 & 0.67 & 11.96 & 0.42 & 0.55 & 13.04 & 0.91 \\
\hline
\end{tabular}

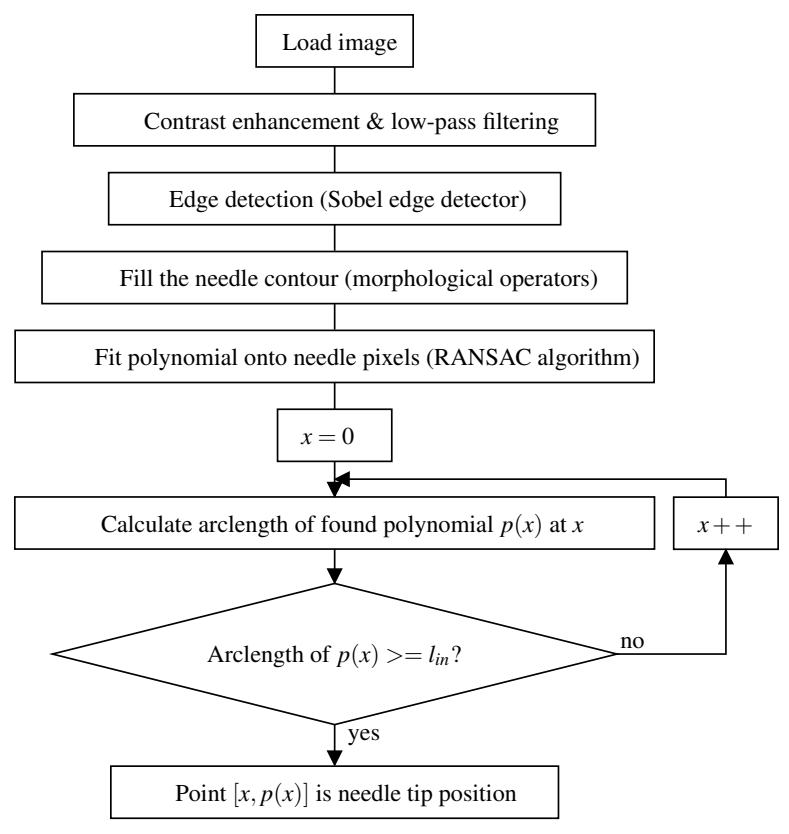

Fig. 4. The needle tip detection algorithm.

The phantom tissue used for the experiments is made from agar of type A360-500 (Fisher Scientific International Inc., Hampton, NH, USA). The stiffness of the agar phantom tissue can be adjusted by the ratio of agar to water used. Two different tissue samples are used. The agar per liter of water for Tissue 1 and Tissue 2 are $45 \mathrm{~g}$ and $22.5 \mathrm{~g}$, respectively. This means that Tissue 1 is stiffer than Tissue 2.

The insertions are performed at two constant insertion velocities of $20 \mathrm{~mm} / \mathrm{s}$ and $60 \mathrm{~mm} / \mathrm{s}$. The insertion depth for all experiments is set to $135 \mathrm{~mm}$ as this is a common insertion depth for prostate brachytherapy procedures. The used needle type is a standard 18-gauge prostate seeding needle of length $200 \mathrm{~mm}$ (Eckert \& Ziegler BEBIG, Inc. Oxford, CT, USA). The material of the needle is stainless steel, which has a Young's modulus $E$ of $200 \mathrm{GPa}$. The needle's area moment of inertia $I$ is $7.86 \times 10^{-14} \mathrm{~m}^{4}$.

Fig. 3(a) to Fig. 3(d) show the tip deflection estimation $\hat{\delta}$ results versus the image-based measured tip deflection $\delta$ for all three models. Each plot contains the results of one tissue-velocity combination. All curves show the mean tip deflection (estimated and measured) for three performed repetitions for each tissue-velocity combination. The deflection
Fig. 5. The needle as a dark shadow with a curve representing the polynomial fit of the needle shape and a cross, which marks the position of the found needle tip.

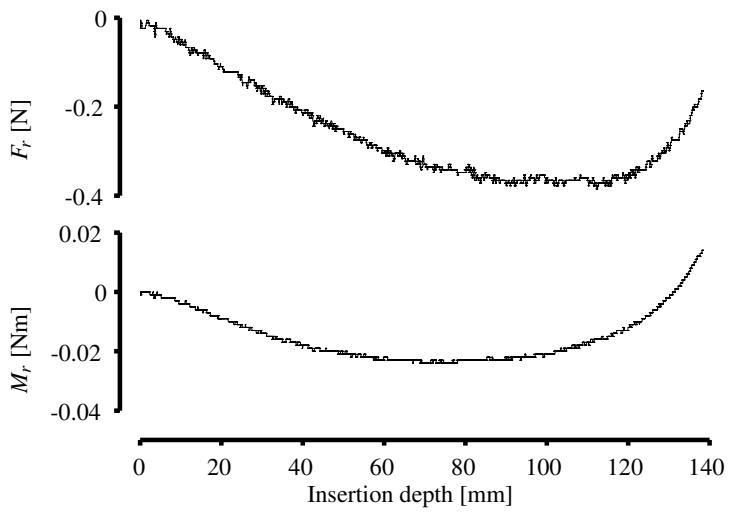

Fig. 6. The lateral force $F_{R}$ and moment $M_{R}$ measured by the fore sensor during insertion into Tissue 2 at an insertion velocity of $20 \mathrm{~mm} / \mathrm{s}$.

data is smoothed by a Savitzky-Golay low-pass filter of order 2 [13]. The plots also show the error between the estimated and the measured tip deflection $\delta-\hat{\delta}$ for each of the three compared models. Table I illustrates the mean absolute error (MAE $=\frac{1}{k} \sum_{i=1}^{k}\left|\delta_{i}-\hat{\delta}_{i}\right|$ ) where $k$ is the amount of samples considered for the MAE. It also shows the mean $|\bar{\delta}|$ of the three trials and the standard error $\sigma / \sqrt{n}$, where $\sigma$ is the standard deviation and $n$ is the number of runs. These parameters are shown for all tissue-velocity combinations and the points where the needle reaches depths of $60 \mathrm{~mm}$ and $120 \mathrm{~mm}$ during insertion. It should be noted that $60 \mathrm{~mm}$ and $120 \mathrm{~mm}$ are not the final insertion depths but chosen depth points during insertion.

\section{A. Discussion}

For deflection estimations up to a depth of $60 \mathrm{~mm}$, all models maintain a low estimation error $(|\delta-\hat{\delta}|<1 \mathrm{~mm})$ except for minor fluctuations, which can be attributed to the remaining data noise. When, however, the insertion exceeds a depth of $60 \mathrm{~mm}$, all models begin to under-estimate the tip deflection. The sole exception is for Tissue 1 and velocity 
$60 \mathrm{~mm} / \mathrm{s}$ where the estimation shows after a depth of $60 \mathrm{~mm}$ the highest precision among all tissue-velocity combinations.

Based on Fig. 3(a) to 3(d), Model 3 performs the best in almost all trials. Especially for Tissue 1, Model 3 shows the best performances with relatively small estimation errors compared to Model 1 and Model 2. The worst performance is shown by Model 1 in all of the trials. This result strongly suggests that our newly developed model (Model 3) represents a significant improvement over the initially proposed model (Model 3) in [9].

As can be observed in Fig 3(a) to Fig. 3(d), at the final insertion depth of $135 \mathrm{~mm}$, the tip deflection is much higher in Tissue 1 than in Tissue 2. The final deflection in Tissue 1 is roughly double as high as in Tissue 2 . This shows that the phantom tissue stiffness is significantly different in between Tissue 1 and Tissue 2. Despite this high variance in tissue properties, Model 3 does not lose precision. This suggests that the model is robust against varying tissue stiffness.

Table I backs up the above observations. The MAE of Model 3 stays well below $1 \mathrm{~mm}$ over all four tissue-velocity combinations for the insertion depth of $60 \mathrm{~mm}$. Although the MAE are very similar for all models throughout the combinations, Model 3 shows the lowest MAE except for the combination Tissue 1 and velocity $60 \mathrm{~mm} / \mathrm{s}$. This remains the case at an insertion depth of $120 \mathrm{~mm}$.

In order to show the consistency of the experimental results across the three trials, the standard errors at depths $60 \mathrm{~mm}$ and $120 \mathrm{~mm}$ are shown in Table I. The low values observed throughout the experiments indicates a high consistency for each tissue-velocity combination.

The reason for the underestimation of tip deflection can be attributed to a change of force distributions along the needle during insertion. An indication of this is given in Fig. 6, which shows the lateral force and moment measured at the needle base by the force/torque sensor. These forces/moments are then used for deflection estimation. Note that at an insertion depth of $60 \mathrm{~mm}$, the force and moment start to flatten out and then change sign. This change in load distributions is not accounted for in the presented models and is a possible reason for the underestimation of deflection using our fixed distributions.

\section{COnclusions And Future Work}

This work introduced an improved approach for sensing the needle tip deflection in real-time during insertion into soft tissue. The only physical sensing device necessary is a force/torque sensor. Furthermore, the approach is independent of explicit a priori characterization of tissue properties. The underlying needle-tissue interaction model and the resulting deflection model were derived and experimentally verified. It was shown that the newly introduced model (Model 3) achieves the best accuracy in estimating needle tip deflection compared to the other two tested models.

In Model 3, a uniform load distribution ( $q_{1}$, see Fig. 1(d)) assumed. In our future work, the investigation of the distributed load shape will be further pursued. A further focus will be the incorporation of friction into the deflection model to improve the accuracy of the proposed model.

Finally, the modelled force $F_{2}$ (see Fig. 1) at the needle tip can be used to estimate the force that the beveled tip enacts onto tissue while cutting into it. Such estimation can be of use for determining tissue properties such as rupture toughness and Young's modulus in real-time.

\section{ACKNOWLEDGEMENT}

The authors would like to thank Jay Carriere of the Telerobotic and Biorobotic Systems Group, University of Alberta for providing an implementation of the RANSAC algorithm.

\section{REFERENCES}

[1] S. Xu, J. Kruecker, P. Guion, N. Glossop, Z. Neeman, P. Choyke, A. K. Singh, and B. J. Wood, "Closed-Loop Control in Fused MRTRUS Image-Guided Prostate Biopsy," in Medical Image Computing and Computer-Assisted Intervention Conference. Springer, 2007, pp. $128-135$.

[2] J. Hong, T. Dohi, M. Hashizume, K. Konishi, and N. Hata, "An Ultrasound-Driven Needle-Insertion Robot for Percutaneous Cholecystostomy," Physics in Medicine and Biology, vol. 49, no. 3, p. 441, 2004.

[3] D. Glozman and M. Shoham, "Image-guided robotic flexible needle steering," IEEE Transactions on Robotics, vol. 23, no. 3, pp. 459-467, 2007.

[4] A. M. Okamura, C. Simone, and M. D. O'Leary, "Force Modeling for Needle Insertion Into Soft Tissue." IEEE Transactions on Bio-Medical Engineering, vol. 51, no. 10, pp. 1707-16, 2004.

[5] S. DiMaio and S. Salcudean, "Needle Steering and Motion Planning in Soft Tissues," IEEE Transactions on Biomedical Engineering, vol. 52, no. 6, pp. 965-974, 2005.

[6] S. Misra, K. Reed, B. Schafer, K. Ramesh, and A. Okamura, "Mechanics of Flexible Needles Robotically Steered through Soft Tissue," The International Journal of Robotics Research, vol. 29, no. 13, pp. 1640-1660, 2010.

[7] M. Abayazid, R. Roesthuis, R. Reilink, and S. Misra, "Integrating Deflection Models and Image Feedback for Real-Time Flexible Needle Steering," IEEE Transactions on Robotics, vol. 29, no. 2, pp. 542-553, 2013.

[8] N. Abolhassani, R. Patel, and F. Ayazi, "Minimization of needle deflection in robot-assisted percutaneous therapy," Int J Med Robot, vol. 3, no. 2, pp. 140-8, 2007.

[9] T. Lehmann, M. Tavakoli, N. Usmani, and R. Sloboda, "Force-SensorBased Estimation of Needle Tip Deflection in Brachytherapy," Journal of Sensors, vol. 2013, 2013.

[10] J. Gere and B. Goodno, Mechanics of Materials. Boston, MA, USA Cengage Learning, 2008.

[11] R. C. Gonzalez and R. E. Woods, Digital Image Processing, 2nd ed. Boston, MA, USA: Addison-Wesley Longman Publishing Co., Inc., 2001.

[12] M. A. Fischler and R. C. Bolles, "Random Sample Consensus: A Paradigm for Model Fitting with Applications to Image Analysis and Automated Cartography," Commun. ACM, vol. 24, no. 6, pp. 381-395, 1981.

[13] A. Savitzky and M. J. E. Golay, "Smoothing and Differentiation of Data by Simplified Least Squares Procedures." Analytical Chemistry, vol. 36, no. 8, pp. 1627-1639, 1964. 